\title{
La ciudad informacional, un site para el arte del siglo XXI
}

\author{
The informational city, \\ a site for the art of the 21st century \\ NATIVIDAd NaVAlón Blesa \\ Universidad Politécnica de Valencia \\ natividad.navalon@gmail.com \\ David TRujILlo RuIz \\ Universidad Miguel Hernández \\ davidtrujillo@gmail.com
}

Recibido: 10/03/2017

Aceptado: 29/03/2017

\section{Resumen}

La ciudad contemporánea está en proceso de transformación por la inclusión masiva de tecnologías y dispositivos inalámbricos de comunicación. La información, los datos y el acceso a éstos, fluye entre los individuos (usuarios) en tránsito, modificando el espacio de interacción, las relaciones sociales y la forma de comportarnos en las nuevas ciudades. Y es que al entorno físico, se le suma una multiplicidad de capas invisibles, donde los flujos de datos (ondas hertzianas) están conectando a dispositivos y personas, formando un nuevo espacio informacional, donde conviven además, las ondas naturales y la contaminación electromagnética. El arte corre paralelo a estas innovaciones tecnológicas e informacionales y se aproxima a este espacio hertziano para evidenciarlo, conceptualizarlo y traducirlo, mostrando sus límites, funcionamientos y peligros. Pero sobre todo, reivindica dicho espacio como un lugar de actuación para determinadas prácticas artísticas. 


\title{
Palabras clave
}

Espacio hertziano, arte, ciudad, comunicación, dispositivos inalámbricos.

\begin{abstract}
The contemporary city is undergoing a process of transformation due to the massive inclusion of wireless communication technologies and devices. Information, data and access to these flow among the individuals (users) in motion, modifying the space of interaction, social relations and the way we behave in new cities. On top of this, a multiplicity of invisible layers, where the data flows (hertzian waves) are connecting devices and people, is being added to the physical environment, thus forming a new informational space, where natural waves and electromagnetic pollution coexist. Art is running parallel to these technological and informational innovations and approaches this Herzian space to evidence it, to conceptualize it and to translate it, showing its limits, functions and dangers. But above all, it reclaims that space as a place of action for certain artistic practices.
\end{abstract}

\section{Keywords}

Hertzian Space, art, city, communication, wireless devices.

Referencia normalizada: NAVALÓN BLESA, NATIVIDAD - TRUJILLO RUIZ, DAVID (2017): "La ciudad informacional, un site para el arte del siglo XXI". Arte y Ciudad. Revista de Investigación, nº 11 (abril), págs. 7-32. Madrid. Grupo de Investigación Arte, Arquitectura y Comunicación en la Ciudad Contemporánea, Universidad Complutense de Madrid.

Sumario: 1.- La ciudad informacional, un site para el arte del siglo XXI. 2.- La ciudad informacional, un concepto en construcción. 3.- El nuevo espacio hertziano, un site para el arte del siglo XXI. 4.- Bibliografía.

\section{La ciudad informacional, un site para el arte del siglo XXI.}

La asimilación gradual de la informática por los sistemas de comunicación (telemática) desde la segunda mitad del siglo $\mathrm{XX}$, con invenciones como el modem o con la aparición de Internet, ha ido transformando la configuración de nuestras ciudades, las formas de relación y comunicación entre las personas, los modelos de producción y consumo, y las estructuras económicas y de 
poder. La liberación del cable, como consecuencia de la actual revolución tecnocientífica en el ámbito de lo inalámbrico, con factores como el abaratamiento de los costes de producción de los dispositivos móviles, ligado al surgimiento de distintas tecnologías y la creación masiva de infraestructuras de redes inalámbricas en las ciudades, entre otros, han permitido el acceso a Internet desde cualquier lugar y hora.

Todo ello ha supuesto una transformación urbana y social, pues ha facilitado, la conexión (online) permanente, y como consecuencia, brindar la capacidad de producción y recepción de contenidos, datos e información que ahora están en constante movimiento. Esta información (datos, imágenes, videos, sonidos y texto) generada, al igual que las señales de telefonía móvil, radio y televisión, viajan a través de ondas radioeléctricas (también llamadas hertzianas) por el espacio. Dichas ondas, atraviesan edificios, personas y objetos, son imperceptibles e invisibles, y están a la espera de ser reveladas y traducidas por los usuarios mediante los dispositivos.

Dicho espacio ha pasado, de ser sólo una preocupación de científicos, ingenieros de telecomunicaciones, organismos reguladores (UIT) y empresas de telefonía móvil y otras afines, a inquietar y afectar directamente a toda la población. $\mathrm{Y}$ es que, en un día cualquiera, una persona consciente e inconscientemente puede llegar a utilizar multitud de sistemas basados en las tecnologías inalámbricas. Entre las más habituales encontraríamos la radio, la televisión, la telefonía móvil y el acceso a Internet vía Wi-Fi, ya interiorizadas por la sociedad, pero hay muchas más, el Bluetooth, el NFC (tarjetas de acceso a la habitación de un hotel, pago con tarjetas, billetes de tren, etc.) o el RFID (el telepago Vía-T de las autopistas, seguimiento de paquetes, etiquetas de seguridad de la ropa, etc.). Y esto es sólo el principio, como vaticinan los teóricos del Internet de las cosas (IoT), ya que se prevé que en 2020, habrá más de 50.000 millones de dispositivos conectados.

Nuestra investigación se centra, precisamente, en presentar la evolución del concepto de ciudad informacional, en busca de una ciudad que contenga las tecnologías de comunicación inalámbricas actuales. Destacando por un lado, cómo es y qué características se le pueden atribuir a esta nueva ciudad informacional, y por otro, cómo la utilizan los artistas como site y por consiguiente, los diferentes modos de afrontar dicho espacio. 


\section{La ciudad informacional, un concepto en construcción.}

La ciudad informacional ha ido evolucionado por la introducción de las tecnologías de comunicación electrónica globales y de su infraestructura digital. Para entender dicha evolución y poder adjetivarla, nos vamos a centrar en diferentes aportaciones que, desde múltiples ámbitos de estudio, han influido en estas nuevas tecnologías, así como en su repercusión en el espacio urbano y en el comportamiento del ciudadano usuario $^{1}$ en dicho espacio.

Nuestra selección no es absoluta, pero sí necesaria para entender el proceso de transformación de la ciudad medial, y además nos permite sentar las bases, y completar el concepto de espacio hertziano y de las prácticas artísticas que surgen a partir de este nuevo espacio de actuación. El arte es un reflejo de la sociedad del momento, y no es ajeno, en ningún caso, a la realidad existente. Si las ciudades cambian, el arte también, las preocupaciones e intereses giran en torno a la vida y a las formas de relacionarse y comunicarse entre las personas, en el espacio, en el ciberespacio, y ahora en el espacio hertziano. Este trabajo nos servirá para constatar que la creación artística corre paralela a esas innovaciones tecnológicas e informacionales y sobre todo para evidenciar, como hemos apuntado anteriormente, que este espacio hertziano se convierte en un lugar de actuación para determinadas prácticas artísticas.

Comenzamos por el término Ciberespacio, porque será utilizado por pensadores y artistas para explicar o referenciar las nuevas ciudades. Fue acuñado por el novelista William Gibson en los años 80, bajo la perspectiva de un mundo que comenzaba a conectarse a Internet a través de cable, para explicar el ambiente futurista de su novela Ciberpunk Neuromante, en la que se adelantaba premonitoriamente a la revolución digital y a la ciencia genética (Gibson, 1984):

El ciberespacio. Una alucinación consensual experimentada diariamente por billones de legítimos operadores,... Una representación gráfica de información abstraída de los bancos de todos los ordenadores del sistema humano. Una complejidad inimaginable. Líneas de luz clasificadas en el no-espacio de la mente, conglomerados y constelaciones de información. Como las luces de una ciudad que se aleja.

\footnotetext{
${ }^{1}$ En sentido general, un usuario es el conjunto de permisos y de recursos (o dispositivos) a los cuales se tiene acceso. Puede ser tanto una persona como una máquina, un programa, etc.
} 
El término fue matizado por Bruce Sterling, una década más tarde, en la introducción de La caza de hackers. Ley y desorden en la frontera electrónica donde escribe (Sterling, 1992):

Desde la década de los 60, el mundo del teléfono se ha entremezclado con las computadoras y la televisión. $\mathrm{Y}$ aunque no hay materia aún en el ciberespacio, nada que puedas manejar, tiene ahora una extraña clase de corporeidad. Hoy en día tiene sentido hablar del ciberespacio, como de un lugar. Porque ahora la gente vive en él. No unas pocas personas, no sólo unos pocos técnicos y algunos excéntricos, sino miles de personas, personas corrientes. Y no durante poco rato, sino durante horas a lo largo de semanas, meses, años. El ciberespacio es hoy en día una Red, una Matriz de alcance internacional, que crece rápida y constantemente. Crece en tamaño, en riqueza y en importancia política.

Sterling da un punto de vista básico al introducir el concepto de lugar para definir el ciberespacio, siendo determinante para la creación artística, porque desde que surgió Internet, no se han dejado de realizar piezas específicas para dicho lugar intangible, sobre todo desde el Net.art. Señalaremos en este ámbito las prácticas activistas (Mark Napier, Muntadas, Heath Bunting,...); la programación pura o software art (Adrian Ward, Radical Software Group, ...), los juegos (Cory Arcangel, Natalie Bookchin, ...) o la exploración de las posibilidades que este medio ofrece, como el Hypermedia Art (David Blair, Claude Closky, Garnet Hertz,...), entre otras. Muchas de estas prácticas se extenderán en el espacio hertziano al acceder al ciberespacio sin cables.

Siete años después, Pierre Lèvy también busca una definición a lo largo de su obra ¿Qué es lo virtual?, y amplía las características del ciberespacio añadiendo el acceso desde cualquier punto del planeta, donde se mezcla la unidad, la localización y la identidad, con la inteligencia colectiva y donde define el ciberespacio como “...un espacio de transacción cualitativamente distinto, en el que los roles respectivos de los consumidores, los productores y los intermediarios, se transforman profundamente." (Levy, 1999). Para Levy, en dicho espacio cada usuario es potencialmente el emisor y el receptor, el productor y el consumidor de datos. Además, las relaciones de dichos usuarios se realizan por intereses y gustos comunes.

Muchas de las afirmaciones propuestas por Levy influirán en el arte contemporáneo, sobre todo por la participación activa del espectador en la cons- 
trucción o lectura de la obras con sus propios dispositivos, ya que en ocasiones los datos producidos por sus terminales crearán la obra, y en otras, los necesitarán para visualizar y revelar o completar las piezas de los artistas. Un ejemplo de ello es la obra Mimmi (2014), que reaccionaba con cambios de color según el estado anímico de la ciudad, en base a la información volcada por los ciudadanos de la misma vía Twitter.

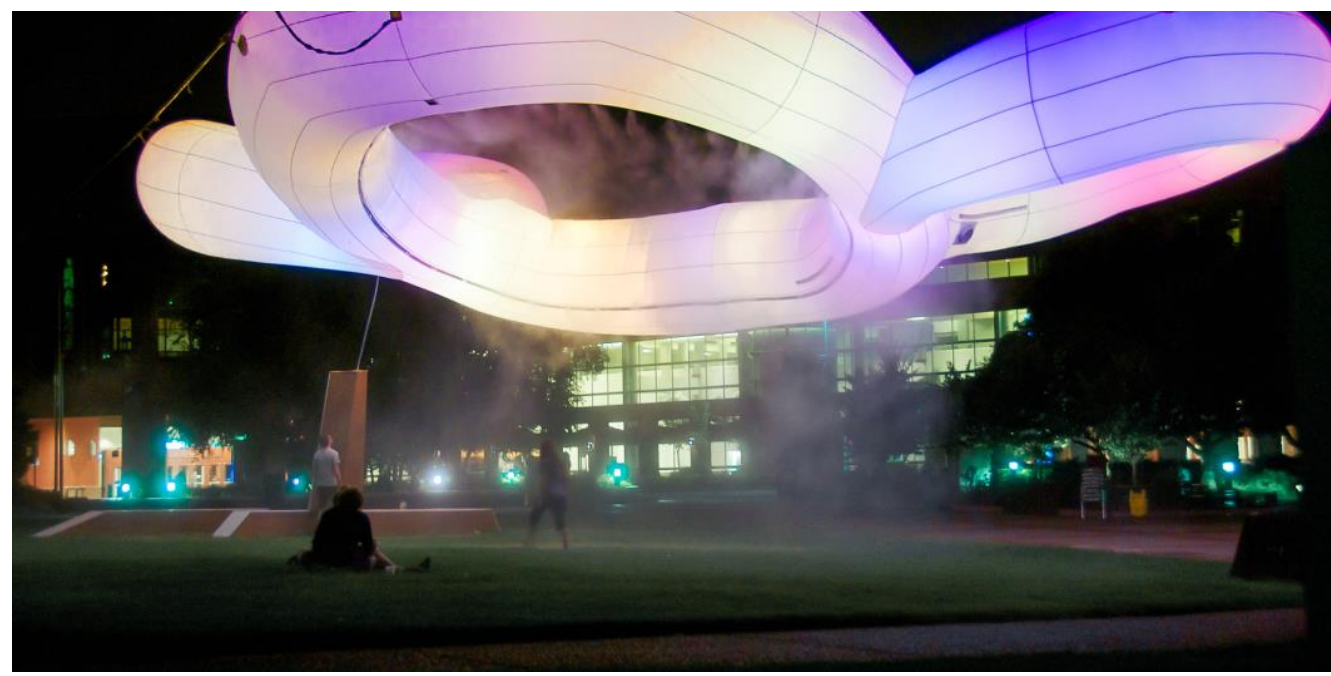

Fig. 1. Mimmi, 2014. Urbain Drc. Extraída de la web: https://creators.vice.com/

Paralelamente y desde la realidad (no virtual pero si medial), surgieron términos para comprender las nuevas ciudades que estaban apareciendo por la evolución de los medios de comunicación globales y personales. Así, Paul Virilio presentó la Ciudad Sobreexpuesta (Virilio, 1991), como una ciudad radicalmente intensa y dinámica, cuyo aspecto es continuamente reconstruido por las pantallas electrónicas, donde el tiempo cronológico e histórico, da paso al tiempo real de la pantalla del ordenador y el televisor, coexistiendo todo ello de manera instantánea.

La ciudad de Virilio, además, es ajena a la identidad y a la memoria colectiva de los lugares, con el tiempo real aquellos se convierten en intercambiables, lo que genera una distopía de donde han sido erradicadas ciudades y lugares geográficos. Es decir, la desaparición del espacio real va en paralelo a la desaparición del tiempo local o histórico o, como dice este autor, la “urba- 
nización del espacio real" da paso a la "urbanización en tiempo real", una nueva forma de crear la ciudad basada en las lógicas informáticas y televisivas. En esta metrópolis se producen interrupciones en el aspecto físico, agujeros electrónicos donde una infinita transparencia sustituye a la materia arquitectónica. $\mathrm{Y}$ donde las simulaciones que generan se intercalan con el espacio urbano, erosionando sus límites y convirtiéndolo en algo permeable que tiende a desaparecer, como dice el propio Virilio, su persistencia es sólo retinal.

Hay que destacar que esta visión la realiza cuando todavía las comunicaciones digitales inalámbricas no estaban implementadas como en la actualidad, y aún así, nos presenta algunos aspectos y cualidades de la ciudad actual, como la desmaterialización, la transformación del tiempo cronológico a uno instantáneo y la permeabilidad a las corrientes de información.

Dos años más tarde, en 1993, Toyo Ito en su escrito Un jardín de microchips (La imagen de la arquitectura en la era de la microelectrónica), nos presentó la ciudad contemporánea a través de una imagen (un microchip) utilizada como metáfora para destacar las características comunes de la ciudad con el microchip. Un espacio generado por áreas no visibles en el que conviven la parte física (agua, viento, personas, edificios, etc.) y la fenomenalidad de la nueva ciudad (información compartida, infinidad de aplicaciones y flujos electrónicos).

En su libro, nos habla del aumento de los flujos de datos invisibles que crearán una arquitectura como artificio para retener y transmitir la información, dominando el espacio urbano real (Ito, 1993):

(..) la ciudad como fenómeno es, después de todo, un espacio con una función (efecto) efímera generado por el flujo electrónico invisible y no toma una expresión morfológica. La ciudad como fenómeno transforma la superficie de la ciudad real en una ciudad de ilusión cubierta por la luz, los sonidos, las imágenes y los signos.... Nosotros no podemos plasmar este espacio de informaciones en imágenes visibles ya que no forman una red física, pudiéndose apreciarlas sólo a través de los terminales.

La propia negación de Ito sobre la no posibilidad de visualizar esos flujos invisibles, se pone en entredicho con la aparición de nuevas tecnologías. Ito destaca la necesidad de revelar, visualizar y entender cómo funcionan esos flujos invisibles, porque influyen, y cada vez más, en nuestra vida y arquitectura. Esta necesidad de visualización de los flujos da pie a nuevas manifesta- 
ciones artísticas, precisamente lo vemos con un sentido activista en la obra de Michelle Teran, life a user's manual (2003), quien equipada con un antena de 2,4Ghz y televisores de tubo, captaba las señales de las cámaras inalámbricas de videovigilancia domésticas en recorridos por las ciudades, y las mostraba en video a tiempo real, comprobando que los límites de la realidad física, no coinciden con los del espacio hertziano.

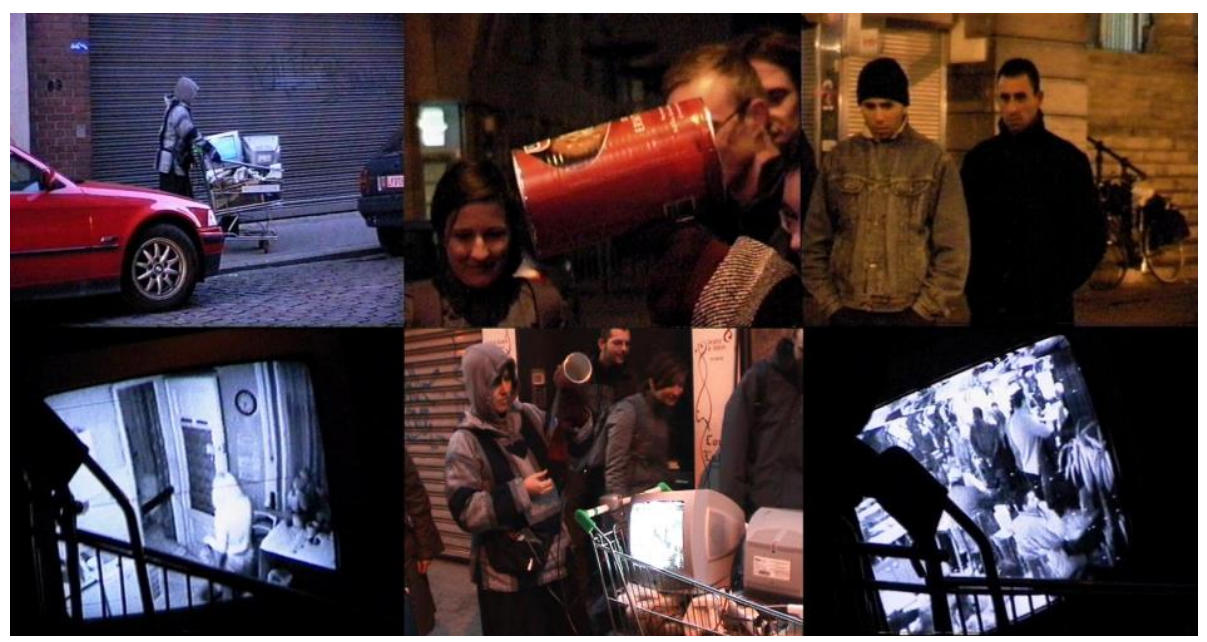

Fig. 2. Life a user's manual, 2003. Michelle Teran.

Extraída de la web del autor: http://www.ubermatic.org/?p=221

Es indudable la influencia de la teorías de Ito en el ámbito de la creación, vemos por un lado, su influjo en los propios arquitectos, tanto en la manera de conceptualizar los espacios como en los propios materiales usados, más sensibles, etéreos, con memoria digital y configurables a razón de las nuevas necesidades informacionales y sensitivas de los ciudadanos. Y por otro lado, su influencia en las prácticas artísticas que usan el espacio como site, con instalaciones e intervenciones híbridas (espacio físico+virtual), interactivas, relacionales (tanto con los espectadores como con otros espacios), sensibles, vivas, a través de las comunicaciones y las redes. Podemos ver la obra de Rafael LozanoHemmer, Alzado vectorial (1999), donde conecta a espectadores de todo el mundo vía Internet, haciéndoles además partícipes de la obra final, posibilitando la creación del diseño de los haces de luz, que fueron proyectados en la ciudad, uniendo los dos espacios físicos (donde se encontraban los usuarios de la web y la ciudad donde se realizaba la exposición final), a través de uno virtual. 
Fig. 3. Alzado Vectorial, 1999. Rafael Lozano-Hemmer. Extraída de la web del autor: http://www.lozanohemmer.com/vectorial_elevation.php
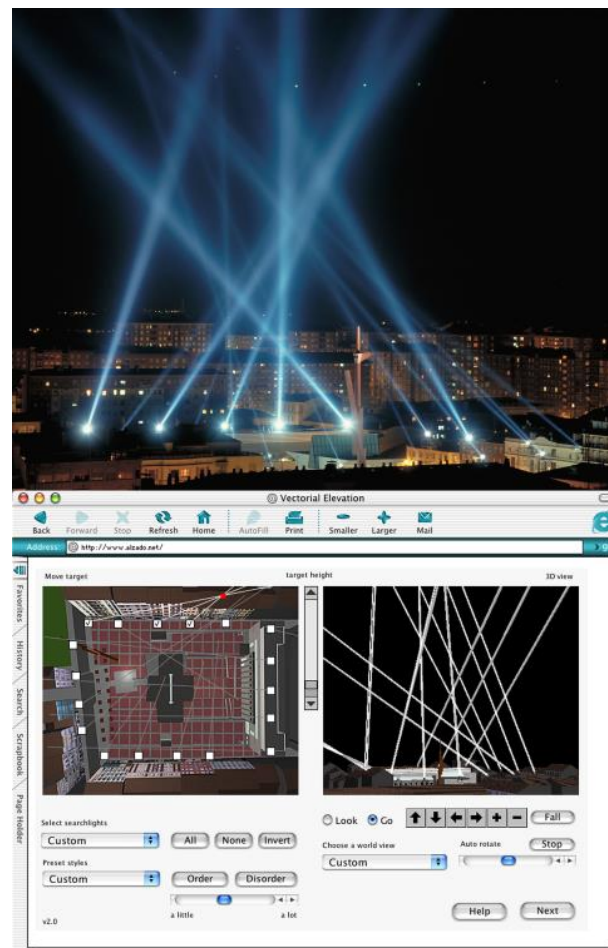

Otra de las aportaciones fundamentales para la configuración de la ciudad, se la debemos a Marcos Novak, que en su búsqueda de ir más allá en la relación entre la arquitectura y la evolución tecnológica de los años 80, acuñó el término Transarquitectura para referirse a la arquitectura de la transmodernidad, en un intento por proveer una vía de discusión entre la telemática y las posibilidades arquitectónicas que se presentaban. Definición similar a lo que Virilio denominó capa permeable en el entorno urbano, pero con el añadido de la aparición de la red Internet y los avances en la computación. Así, el significado del término de Novak evolucionó hacia una arquitectura interesada en comprender el ciberespacio, su construcción digital por software y datos, y relacionada con los nuevos habitantes, los usuarios o sus avatares.

Pero el término que nos interesa de Novak es la Arquitectura líquida, puesto que es una acualización de la transarquitectura: "una arquitectura líquida que existe en el ciberespacio y es transmitida por las redes de información global y otra que existe como un doble electrónico e invisible que se superpone a nuestro mundo material" (Novak, 1993). 
Así, estableció una poética del Ciberespacio y de las posibilidades de los algoritmos en las artes. Novak propuso un espacio ilimitado que permitía desarrollar espacialidades no euclidianas: la cerogravedad, el no lugar, la no identidad, que sólo son posibles en la virtualidad. Además, esta arquitectura líquida tiene la capacidad de ser mutable y maleable, aplicándose al tiempo y a las necesidades espaciales, ya que los sitios no definen de antemano las posibles necesidades de los usuarios, sino que se adaptan a ellas. Este concepto influirá en la definición de La ciudad de Bits de Mitchell y en la Arquitectura de código abierto de Haque, las cuales veremos más adelante.

La ciudad de Bits que William J. Mitchell plantea (Mitchell, 1995), nos ofrece una ciudad conectada, en la que las actividades económicas, sociales y culturales se desarrollan en el ciberespacio. Este autor la define como un ente construido virtualmente, no arraigado en ningún lugar geográfico concreto $\mathrm{y}$ habitado por descorporeizados cibernautas. Donde la parte electrónica, digital y virtual ha desplazado a la física y las construcciones se han transformado en software, en programas que almacenan los bits de información y que el edificio necesita para ejercer sus nuevas funciones.

Además, nos plantea un cambio en la aportación de los arquitectos: en una nueva ciudad, en la que los habitantes se están convirtiendo en Cyborgs, los edificios perderán su claridad y su estatus, ya que los usos del espacio edificado ya no son asignados de manera permanente y dependen del minuto a minuto del software y el flujo de bits, siendo reconfigurables y transformables según los intereses de los usuarios.

Este autor imaginó edificios que no encajan en ninguna tipología arquitectónica convencional, tanto social como estructural, donde los accesos y los recorridos interiores se convierten en conexiones electrónicas, y las fachadas han sido sustituidas por gráficos de pantalla donde se exponen y venden productos, el equivalente a los antiguos escaparates y espacios de venta: "La fachada no debe ser construida de piedra, sino de píxeles en miles de pantallas repartidas por todo el mundo" (Mitchell, 1995). Esta aseveración nos recuerda a la ciudad sobreexpuesta de Virilio, pero añade la conexión. En dicho sentido, desde la arquitectura y el arte, se están teniendo en cuenta estos planteamientos, ya que cada vez más surgen construcciones donde el exterior del edificio está formado por sistemas de representación de imágenes en movi- 
miento configuradas a tiempo real, como el Bix Electronic Skin en Graz (Austria), la Galleria Hall West, en Seul (Korea) o la fachada digital del Medialab Prado en Madrid (España), entre otros muchos.

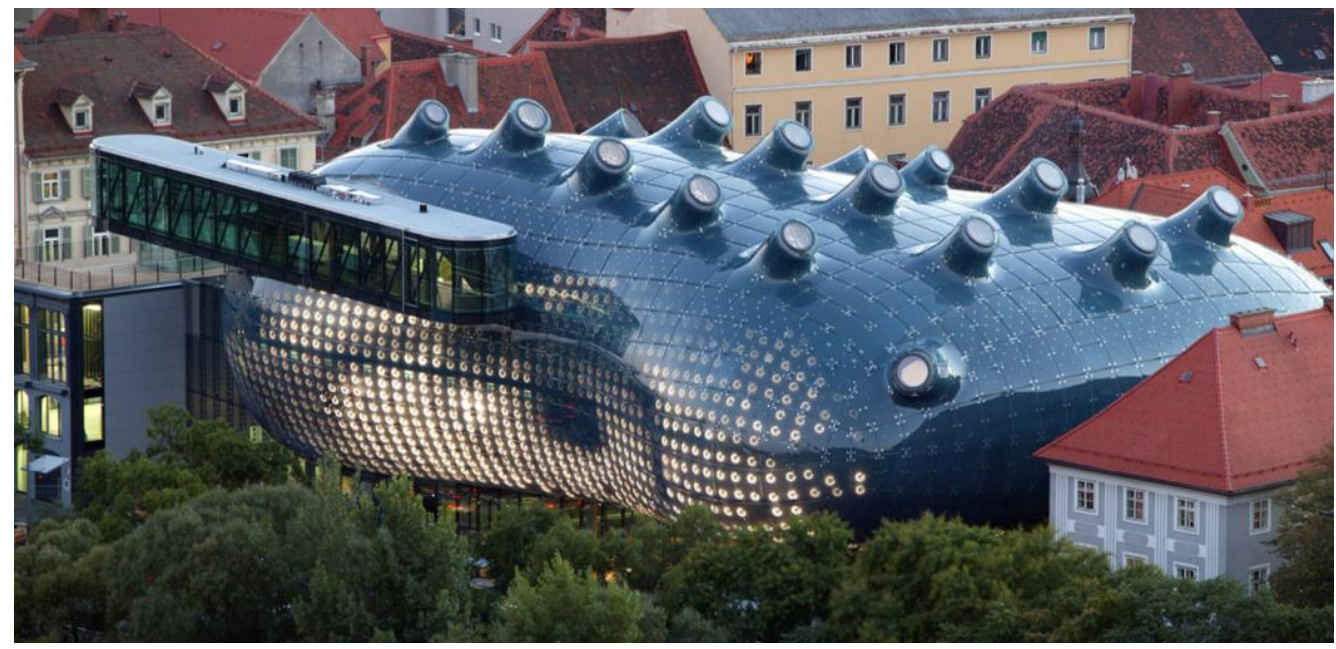

Fig. 4. Bix Electronic Skin en Graz, Austria.

https://www.moma.org/interactives/exhibitions/2011/talktome/objects/133767/

Mitchell analizó esta nueva infraestructura y sus implicaciones para la vida cotidiana, y propuso nuevas estrategias para la creación de ciudades que no sólo fueran sostenibles, sino que tuvieran sentido desde el punto de vista económico, social y cultural en un mundo informacional. Así pues, plantea las Smart Cities, donde todos los dispositivos electrónicos de uso cotidiano, así como los espacios arquitectónicos, tendrán sistemas de inteligencia incorporados y estarán interconectados (Internet de las cosas). Dicha interconexión está posibilitando a los artistas el poder extraer datos y patrones de objetos y personas; donde la investigación sobre su posicionamiento lleva implícito un seguimiento y control. El resultado de estos estudios se verá reflejado en sus obras. La obra TrashTrack (2009) es un ejemplo de ello, puesto que al introducir pequeñas tarjetas geolocalizadoras en objetos tras su desuso, reflejaban su posicionamiento a tiempo real, el recorrido de éstos a través del sistema de eliminación de basuras, mostrando rutas ilógicas y procesos poco eficientes, que de otro modo seguirían ocultos.

Desde la sociología, nos encontramos una de las reflexiones más referenciadas sobre el nacimiento de un nuevo espacio influenciado por las tecnologías 
de la información y comunicación, como fue el análisis de Manuel Castells englobado bajo el concepto de La sociedad red (Castells, 1996). En este estudio planteó una sociedad influenciada por la tecnología e Internet, una sociedad que gestiona, produce y se organiza en torno a redes de información electrónicas. Así para Castells, la sociedad red sería una combinación de la globalización capitalista y las Tics, con unas características estructurales basadas en relaciones de producción, de experiencia y de poder, donde la realidad estaría construida por redes productivas de información (inmaterial) que generan, almacenan, procesan, y transmiten información, sustituyendo el espacio físico de los lugares y el tiempo histórico, por el espacio de flujos y los tiempos instantáneo y asincrónico.

Precisamente, el Espacio de flujos fue una de sus aportaciones fundamentales, pues es el lugar donde operan y funcionan las interacciones informacionales de la sociedad red. Dicho espacio se puede describir (no definir), como combinación de al menos tres capas (Castells, 1996):

La primera capa, el primer soporte material del espacio de los flujos está formada por un circuito de impulsos electrónicos. La segunda capa la forman sus nodos y ejes; y la tercera capa, es la organización espacial de las elites gestoras dominantes (más que clases): son las que organizan, gestionan y articulan ese espacio.

Sobre la realidad digital de la ciudad, encontramos la aportación de Javier Echeverría con el Tercer entorno (Echeverría, 1999), donde propone la existencia de un lugar bajo el control de las tecnologías de la información y de la comunicación. Echeverría nos presenta una realidad de flujos, informacional, electrónica, donde la distancia pasa a ser irrelevante, y donde la interacción se realiza a través de las redes telemáticas, sin necesidad de estar presente. Además, advierte que la arquitectura y el urbanismo tienen que adaptarse a los cambios producidos en el tercer entorno, como condición previa, para poder utilizarlo. De no hacerse, la ciudad quedará excluida de los flujos y no existirá.

Queremos destacar que Echeverría propone la existencia de unas élites que denomina "los señores del aire", quienes dirigen, controlan y luchan por las redes de la telepolis (Echeverría, 2001). Las preguntas que propone Echeverría son tratadas por artistas que utilizan el espacio hertziano como lugar de actuación, y que hemos denominado activistas (por su herencia del Net.art). 
Artistas que buscan respuestas sobre el funcionamiento de los flujos y sus dueños, y las influencias de poder y control sobre la población, con piezas que sabotean redes o creando las suyas propias libres. Una de estas obras fue Wifi Bedouin (2004), de Julian Bleecker, una estación wifi portátil que proporcionaba a las personas que se encontraban cerca de él, acceso a un falso Internet y a varias aplicaciones preestablecidas como el chat o el blog.

Por su parte, José Pérez de Lama presenta las Geografías de_la_multitud [conectada] (2003), donde analiza la confluencia de la arquitectura con la tecnología, el arte y el activismo. Lama, influenciado por la teoría de Castells sobre los flujos y lo temporal, nos presenta una arquitectura (la de la sociedad de la información) que tiene que transformarse o dejar camino a otras áreas de conocimiento para que se hagan cargo. Con un razonamiento simple propone que, si el espacio está cambiando hacia uno tecnológico e informacional, la arquitectura tiene que evolucionar y adaptarse utilizando materiales compatibles tecnológicamente, en caso contrario, otros ámbitos de estudio más comunicacionales asumirán las tareas de conocer, interpretar, organizar, y producir los nuevos habitares. Esta arquitectura debe pensar antes en los flujos de información que en los espacios físicos, y debe sustituir las características de los viejos lugares como la identidad, hegemonía, jerarquía y espectáculo por características de lo digital en red (hibridación, pluralidad, horizontalidad y performance).

Pero lo que más nos interesa de la teoría de Pérez de Lama, es su visión de otro rizoma de flujos, denominado Geografías de la multitud conectada (Pérez de Lama, 2003), que comparte el espacio informacional con el poder (capitalismo global), con el "imperio" o con "los señores del aire" vistos anteriormente, pero que a diferencia de éstos, está al servicio de la comunidad. Para Lama son un conjunto de resistencias, fuerzas creativas de la multitud, en las que encontraríamos artistas, activistas, hackers, arquitectos, que mediante los propios sistemas mediales de los flujos, sus redes, tecnologías, herramientas digitales se apropian del espacio urbano como un nodo de los espacios de flujos, para rediseñar el territorio colectivo.

Estas Geografías de la multitud, no pretenden luchar u oponerse contra los flujos (como hablaba Castells con la identidad y lo local), pero sí lo hacen contra las estructuras de poder. 
Todos los trabajos artísticos a partir de dichas teorías, los podemos englobar dentro del concepto activista, junto con los Smart mobs². Trabajos como los realizados por el propio Pérez de Lama en su grupo Hackitectura.net, que junto a Pablo de Soto y Sergio Moreno intentan luchar contras los flujos hegemónicos y los lobbies telecomunicacionales.

Aunque ya hemos visto anteriormente autores que plantean una arquitectura fluida, liviana, transparente y llena de información digital (Virilio, Ito, Mitchell, entre otros), queremos destacar la propuesta que en 2005 hace Usman Haque (arquitecto de formación) de su visión de Espacio blando frente a la arquitectura dura, porque introduce los campos electromagnéticos y las ondas como material artístico y arquitectónico.

$\mathrm{Y}$ es que propone, frente al hardspace (hierro, cemento, cristal etc.), una realidad fluida, el softspace, un mapa invisible superpuesto, una serie de elementos dinámicos como sonidos, olores, ventilación, temperaturas que contribuyen, tanto o más que el hardspace, para crear la experiencia de un edificio o de un espacio. Además en el softspace, intervienen los campos electromagnéticos y las ondas de radio. Como apuntó el propio Haque en su texto para artfutura (Haque, 2005):

A través del desarrollo de la interfaz han explorado lo que podrían denominarse las tecnologías de los "espacios blandos": sistemas que incorporan las cualidades efímeras de la arquitectura incluyendo el olor, el sonido, la luz, el calor y los campos electromagnéticos. Esta propuesta se concentra en las interacciones que forman la experiencia del espacio y proponen sistemas que afectan estas interacciones.

El propio Haque, es uno de los creativos más destacados en la realización de piezas que tratan los espacios blandos, y en algunas ocasiones (como consecuencia lógica y por extensión con la utilización de las ondas), los hertzianos. En la imagen podemos ver la obra Sky Ear (2004), una escultura flotante de globos de helio que cambiaba de color al traducir las señales radioeléctricas de las redes de telefonía móvil a su paso por las ciudades.

\footnotetext{
${ }^{2}$ Movimientos sociales o políticos a través del teléfono móvil o mensajes de texto. Smart Mobs se define como una multitud inteligente, es una forma de organización social que nace y se estructura a través de las nuevas tecnologías de la información y las comunicaciones, para realizar una tarea coordinada.
} 
Fig. 5. Sky Ear, 2004. Usman Haque. Extraída de la web del autor: http://www.haqu e.co.uk/skyear/in formation.html

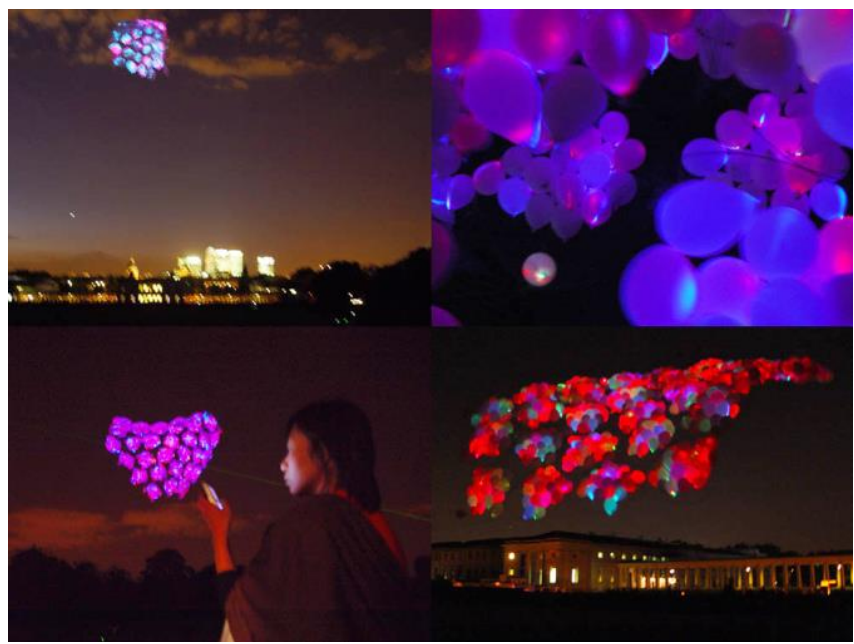

Relacionado con los nuevos medios de comunicación (inalámbricos) y con las tecnologías y dispositivos móviles con acceso a Internet, podemos señalar el territorio informacional de André Lemos (Lemos, 2008). Un espacio híbrido, aumentado, compuesto por flujos, donde lo real y lo virtual se cruzan, y donde múltiples espacios locales se mezclan cuando nos adherimos a la red (en movilidad), interconectando los lugares desde donde consumimos y producimos información. Una realidad, que no se puede separar del ciberespacio, puesto que al estar conectados permanentemente, no podemos separar lo online de lo offline. Este autor señala que lo que define a las nuevas redes es la capacidad de los usuarios de producir contenidos en movilidad (hiperlocalización de la información), consumiendo, produciendo e intercambiando información en forma de datos, mientras transitan, caminan o viajan por las ciudades, o entre ellas.

A partir de estas aportaciones sobre la ciudad informacional, hemos podido extraer dos posturas teóricas enfrentadas. La primera nos advierte de los peligros de esta ciudad: de su velocidad (Virilio), del control por el siempre conectados (Castells), de la privacidad de nuestra información volcada en el flujo de datos, de los intereses ocultos detrás de las multinacionales de la telecomunicación y los gobiernos (Echeverria), etc. La segunda, por el contrario, destaca los beneficios de una ciudad informacional: con una arquitectura cambiante, adaptable a nuestros deseos (Ito, Novak, Haque y Mitchell), o de la participación global a través de los propios sistemas mediales, flujos, redes, 
tecnologías, herramientas digitales para rediseñar el territorio colectivo y hacerlo más público (Pérez de Lama y Lemos).

Dos caras de la misma moneda, más si cabe, si atendemos a que todas ellas afirman la existencia de algo imperceptible ahí fuera, que no se ve, pero que es tan determinante en nuestras vidas como los propios materiales sólidos. Un fluir de información en forma de ondas, que no queda en la imaginación del ciberespacio, sino que se relaciona con el espacio físico (adaptándose o atravesándolo).

Como hemos podido comprobar, el arte se ha hecho eco de todas esas transformaciones urbanas, utilizando las mismas tecnologías de comunicación que iban modificando la propia ciudad, con creaciones en el ciberespacio, en los espacios híbridos y en el espacio invadido por ondas hertzianas. Creaciones artísticas que abarcan preocupaciones conceptuales, reflexivas y didácticas, con obras que revelan los procesos que ocurren en cada uno de los espacios, señalando los límites y peligros; piezas generadas de forma colaborativa a través de las redes, o aquellas que subvierten el funcionamiento habitual de los flujos de información.

A partir de esta exposición creemos necesario una revisión de la ciudad informacional actual, la ciudad hertziana, donde revisaremos el nuevo espacio hertziano convertido en site de determinadas prácticas artísticas del siglo XXI.

\section{El nuevo espacio hertziano, un site para el arte del siglo XXI.}

La ciudad hertziana influenciada por las tecnologías y dispositivos inalámbricos, aúna las posturas positivistas y aterradoras de las aportaciones teóricas anteriores, y las características comunes de todas ellas: la multiplicidad de capas, el tiempo instantáneo y asincrónico, la desaparición de las distancias, los flujos de información, la creación colectiva de datos e información digital en tránsito, y la posibilidad de una arquitectura mutable, maleable y adaptable, con inteligencia. Pero además incluye el espacio físico, el ciberespacio y el lugar invisible donde viven las ondas, el nuevo espacio hertziano.

El término espacio hertziano, fue acuñado por Anthony Dunne (1999) para describir de manera amplia el lugar de interacción entre los dispositivos electrónicos (que emiten campos electromagnéticos) y las personas. Un espacio híbrido artificialmente construido y en expansión, debido al crecimiento en la 
implantación de los dispositivos tecnológicos y directamente relacionado con el auge de las redes inalámbricas de telecomunicación. Se trataría pues, de un paisaje híbrido conformado por zonas de sombras o puntos sin conexión, zonas de reflexión de las distintas ondas que transitan y puntos calientes de conexión (hotspot), es un espacio que no vemos, en este sentido el autor nos señala:

Mientras el Ciberespacio es una metáfora que espacializa lo que ocurre en los ordenadores distribuidos por el mundo, el espacio de la radio es actual y físico, aunque nuestros sentidos puedan detectar sólo una pequeña parte de él (Dunne, 1999).

Lo interesante de la definición de Dunn es que lo plantea, no sólo como un espacio habitado por ondas de diferentes frecuencias y longitudes que surgen de la relación entre el paisaje natural y artificial, y que fluctúan invisibles a nuestro alrededor, sino que además, incluye las interacciones culturales, usos y experiencias del ciudadano en el espacio físico que comparten.

Para nosotros, el término necesita una actualización para poder incluirlo de una manera más amplia y acertada en la ciudad informacional actual. Para ello, hemos tenido en cuenta los condicionantes tecnológicos actuales como el acceso a Internet (y al ciberespacio) de forma inalámbrica, aspecto que no estaba implantado cuando surgió el término, y también hemos contemplado las ondas radioeléctricas naturales, porque entendemos que influyen en las comunicaciones y porque comparten el espectro generado artificialmente. Así, para nosotros el nuevo espacio hertziano sería: el lugar intangible de interacción entre las tecnologías inalámbricas, las personas y el espacio físico, junto con las radiaciones radioeléctricas naturales y las artificiales de los dispositivos eléctricos/electrónicos. Dicho espacio supone, además, el lugar de transmisión de flujos de información a través de las ondas (imagen, audio, vídeo y datos). Este lugar puede funcionar independientemente a la fisonomía de la propia ciudad o de la naturaleza, o junto a ella, donde las ondas suponen una segunda piel imperceptible que bordea el espacio físico.

Pero, ¿Cómo funciona esta nueva ciudad y su espacio hertziano como site? Para nosotros el site, hace referencia al lugar de intención, donde la función del espacio no se reduce a contener la obra, sino que pasa a formar parte de la obra de arte en sí. En el site, las cualidades arquitectónicas/físicas del mismo, las características más conceptuales, sociales y relacionales ancladas al lugar, 
como la memoria, los usos y la propia actividad que allí ocurre, etc., son el núcleo generador o determinantes en la conceptualización de la propia obra.

Al igual que se han ido añadiendo espacios a la ciudad informacional, los artistas han ido añadiendo distintos lugares de actuación o intervención en el espacio. Han pasado de un site relacionado con un espacio físico: sala, edificio, calle o plaza, o incluso recorridos por la propia ciudad determinantes en el acto artístico (acciones o happenings), a la intervención en un site del ciberespacio (con cable) o del espacio híbrido donde se englobarían todas las prácticas colaborativas o de telepresencia del Net.art; y por último a la apropiación del espacio hertziano como site.

En relación al funcionamiento de la propia ciudad informacional como site, nos hacemos eco de la puntualización que hizo José Iges en la Ciudad Resonante sobre el uso que los artistas hacían de ella:

...los artistas han comprendido la ciudad como un incesante emisor de signos, y sus obras han perseguido, mayoritariamente, atrapar esos signos para transgredirlos o trascenderlos, en un contexto así mismo mediático: el de su difusión en espacios públicos de la propia ciudad, en una sala de conciertos o en el medio radio (Iges, 1997).

De esta manera, los artistas pueden diferenciar distintos modos de uso del propio espacio. En primer lugar, como continente de la obra, es decir, a modo de espacio expositivo, como lugar de intención. Los artistas crean las obras y vuelcan su trabajo en la ciudad.

En segundo lugar, como generador de signos, datos, información o situaciones, a partir del cual los artistas recopilan, capturan, roban, rescatan información para realizar trabajos que posteriormente presentarán en otros lugares, con distintos formatos artísticos: instalaciones, videoarte, documentales, Net.art etc. Las prácticas como veremos son diversas, desde recopilar información (mediciones) de las radiaciones electromagnéticas para alertar de los peligros de dicha contaminación, pasando por la privacidad de los datos que flotan en lo invisible de las redes inalámbricas, hasta las manifestaciones de visualización, sonificación y tangibilización.

Y por último, como lugar de actuación, es decir, el espacio hertziano es el lugar de interacción entre obras y espectadores: en las ciudades hertzianas, 
tanto el espacio físico de interacción, como los datos y contenidos de las redes inalámbricas aportan al artista el material que después traducirá en obras. Así pues, el artista manipula, configura y hace perceptibles los datos informacionales, en muchos casos a tiempo real y en movimiento, y lo muestra en el propio espacio hertziano.

Tenemos que apuntar, que el espacio hertziano, en general, funciona independiente a la fisonomía de la propia ciudad, ya que las ondas se propagan a modo de una segunda piel imperceptible que bordea lo físico, y en ocasiones, lo invisible de los flujos puede anclarse en un punto físico localizado.

Una vez acotado el site specific como ámbito de actuación artístico en la ciudad hertziana, vamos a exponer diferentes modos de afrontar dicho espacio por parte de los artistas.

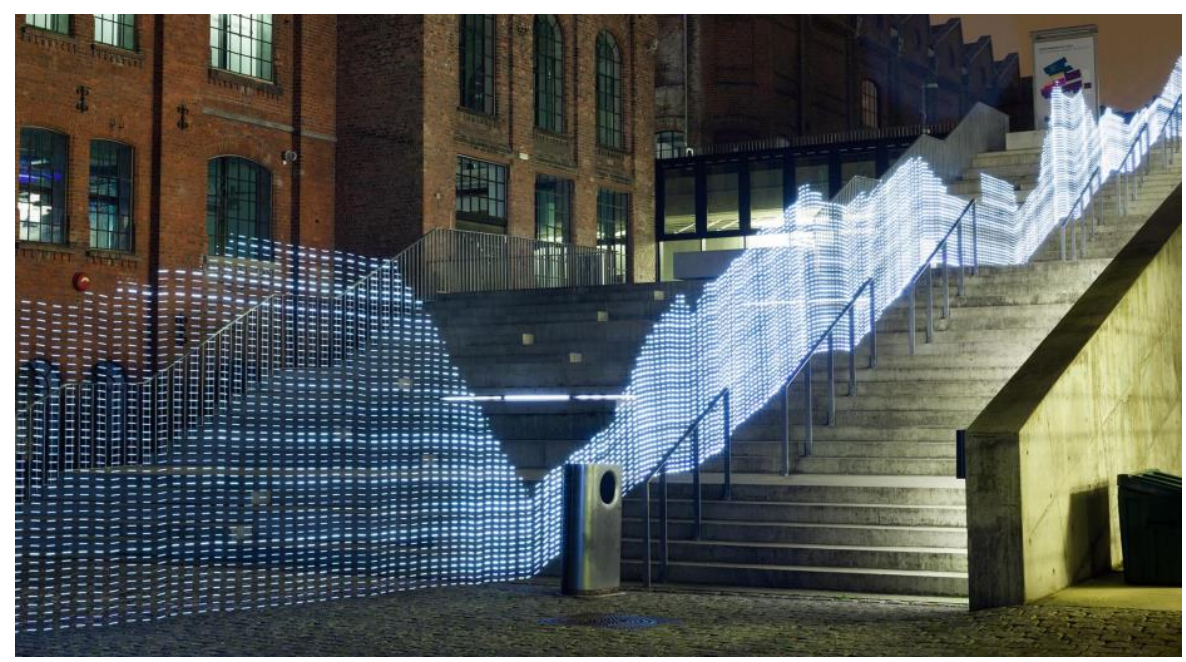

Fig. 6. Light painting Wifi, 2011. Timo Arnall, Jørn Knutsen and. Einar Sneve Martinussen. http://www.elasticspace.com/tags/film

En primer lugar, queremos plantear el trabajo de aquellos creadores que revelan el espacio hertziano. En este ámbito englobamos todas aquellas creaciones artísticas que hacen perceptible el espacio de interacción de cualquier tecnología inalámbrica o aparato electrónico, (nodos, redes, puntos de acceso, intensidad de señal, etc.). En ellas, los artistas evidencian sus contornos, límites, fuerzas, relaciones con el espacio físico, etc. Obras que consiguen traducir dicho espacio invisible a través de lo visual (visualización) con obras como: 
Ghost in the field, Light painting Wifi, Fashionvictims, Invisible forces, Single Pixel Camera Wifi, Mimmi, etc...; a través del sonido (sonificación) con obras como Wifio, Tuning Corridor, CityListeners, Ætherspace, V.L.F. Natural Radio, Hydrogen RT03, etc...; a través de lo audiovisual (visualización + sonificación) donde lo visual se complementa con el sonido: Electrosmog, Frecuencia y Volumen, Very Low frequency fireplace, Wave to Waves to Waves, Ozono, Spektr!, etc; y a través de otros sentidos como el tacto, el gusto y el olor (tangibilización): Sticky Data y Contrataint City.

Por otro lado, recogemos aquellas obras que reflexionan sobre la comunicación inalámbrica teniendo conciencia del espacio hertziano, del espectro radioeléctrico utilizado, y que se centran en los mecanismos, usos y posibilidades de la propia tecnología, desde perspectivas comunicacionales y sociales, más bien neutras o positivas, como en Befote the beep, rome to tripoli o Nearness.

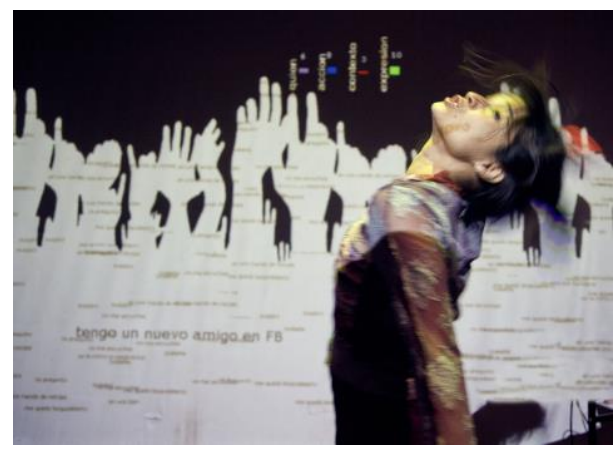

Fig. 7. Before the Beep, 2011. Kònic thtr. http://koniclab.info/es/?project=beforethe-beep-2011/

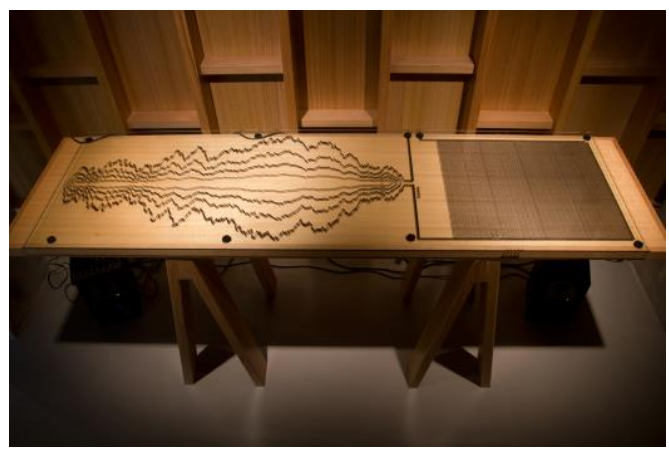

Fig. 8. The SoundWave: Induction drawings. 2012. Haines $\mathcal{E}$ Hinterding.

http://www.haineshinterding.net/2012/01/01 /soundwave-induction-drawings/

También queremos destacar todos aquellos trabajos que hablan sobre los procesos, funcionamiento y características del espectro electromagnético, el comportamiento de las ondas, la propagación de las mismas o sus propiedades, etc. desde un aspecto meramente físico. Con obras como Light bulb, Protrude (Flow) o SoundWave: Induction Drawings.

Una parte de los artistas tienen una posición activista en el espacio hertziano, con obras que se enfrentan a gobiernos y grandes empresas telecomuncacionales y demás reguladores del espectro, para recuperarlo al servicio 
del ciudadano como en: Nodo móvil, Infocalypse Now!, AllFm Tallin, Estonoesinternet, Wifi.Bedouin, Porta2030, etc; prácticas que nos alertan de los peligros de las radiaciones electromagnéticas: Skrunda Signal, Latvian Electromagnetic pollution, Spectrum Survey, Tempest, etc; piezas que nos protegen de los efectos de las radiaciones: Shroud/Chrysalis y Telepathy; e intervenciones que tratan la privacidad y el control de dicho espacio: Life: A user's Manual, X.pose, Spam Tower, Yellow chair, etc.

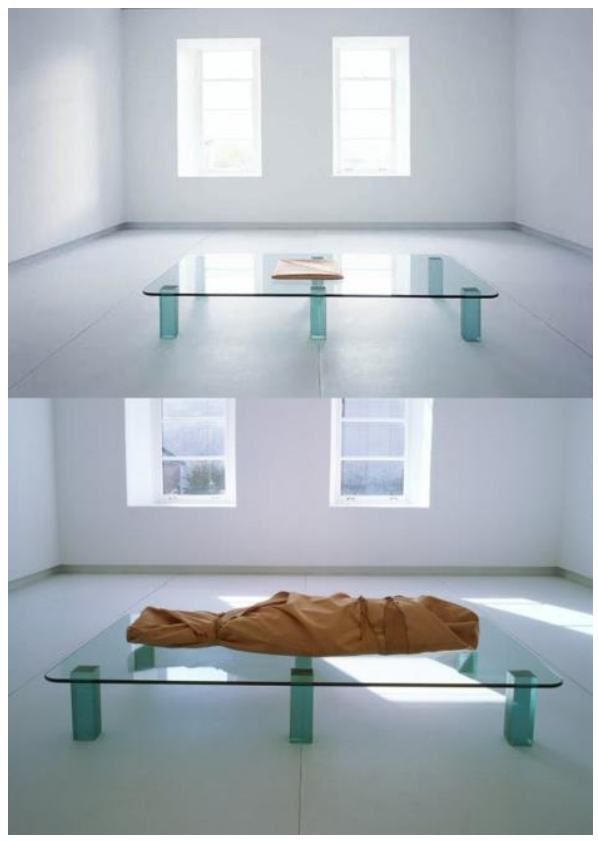

Fig. 9. Shroud/Chrysalis, 2000. Catherine Richards. http://www.fondationlanglois.org/html/e/page.php?NumPage=162

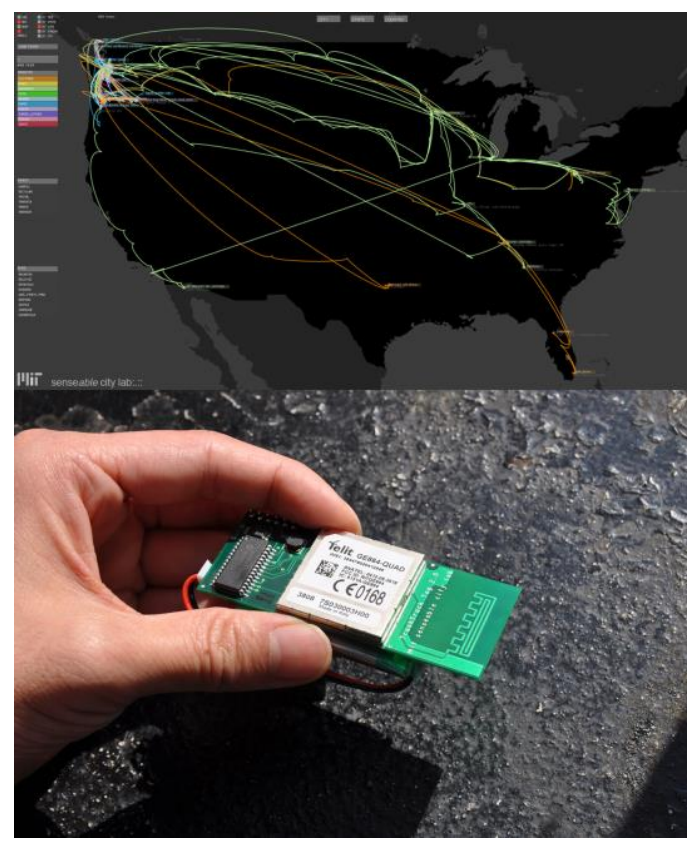

Fig. 10. TrashTrack, 2009. SENSEable City Lab. http://www.jenniferdunnam.com/WasteManagementTrash-Track

Por último, queremos resaltar aquellos trabajos que permiten la localización en el espacio hertziano. Donde la posición de objetos, dispositivos y personas dentro de dicho espacio son determinantes para la génesis de la obra. Así, hemos identificado dos variables. La primera agrupa a aquellas obras que tratan sobre el Internet de las cosas (Iot), y reflexionan sobre la interconexión digital de objetos cotidianos con Internet y con otros objetos: Trapped, TrashTrack o Near Future RFID. En la otra variable, Locative media, están recogidas aquellas obras que hacen referencia a la geolocalización y el posiciona- 
miento, pero en tales prácticas son los dispositivos tecnológicos basados en el GPS los que entran en acción (smartphones, tablets, navegadores, el propio GPS, etc.), y donde a través de estos, la obra toma forma, ya sea activando una aplicación (por el espectador) en un punto localizado (por el artista), o en varios. Ejemplo de ello son los paseos narrativos, como en Las calles habladas y los juegos participativos, como en Can You See Me Now?, Pac-Manhattan, Loc:draw!, Asap, Bioscope, Tactical Sound Garden.

Como podemos comprobar el arte del siglo XXI está conectado de manera natural con la ciencia y la tecnología, porque comparten preocupaciones, temáticas, métodos de investigación y creación, y cómo no, el uso inmediato de las tecnologías como las locativas, de seguimiento o de identificación actuales, que posibilitan nuevas maneras de hacer relacionadas con la ciudad hertziana, nos referimos a las aplicaciones móviles (App), los dispositivos wereables ${ }^{3}$, la deriva y narrativa geolocalizada, juegos o Smart Mobs, entre otras.

Queremos destacar la función didáctica, ideológica y activista de los artistas que se interesan por el espacio hertziano, porque se cuestionan acerca de los supuestos y motivaciones de determinadas tecnologías inalámbricas, mediante la creación de obras que exploran aspectos poco ortodoxos más allá de lo utilitario. Por un lado, buscan revelar al público sus contornos e influencias, su funcionamiento, y por otro, advertir de sus peligros, de las luchas de poder entre las grandes compañías telecomunicacionales y gobiernos por apropiarse de dicho espacio invisible (redes y ondas), de las contaminaciones radioeléctricas y de la privacidad de nuestros datos que flotan y fluyen a través de la ciudad informacional. Obras que nos muestran, entre otras cosas, cómo luchar contra él, creando redes alternativas, saboteando las existentes, o confeccionando habitáculos y ropa especial, para protegernos de las ondas.

Y es que, las tecnologías y dispositivos se inventan y se convierten en productos que quedan tan integrados en nuestras vidas que nadie se cuestiona por qué se inventaron, los problemas culturales que resuelven (o crean) o sus narrativas subyacentes. La introducción de los dispositivos móviles y los sis-

\footnotetext{
${ }^{3}$ Son dispositivos y aparatos tecnológicos, pero éstos están diseñados para ser llevados en alguna parte de nuestro cuerpo (o como complemento de ropa), interactuando con otros sistemas inteligentes como los smartphones.
} 
temas GPS locativos actuales, diluyen la privacidad y el anonimato, primero al introducir el ID personal en los dispositivos y segundo, porque la precisión de localización actual es de centímetros.

Pero mirando un futuro cercano, se abren muchas cuestiones sobre la ciudad informacional además de las locativas: ¿Cómo afectarán la computación ubicua y la conexión inalámbrica global? ¿Será la explotación definitiva, el encadenar a los trabajadores a sus puestos de trabajo donde quiera que vayan? ¿Qué pasará si no tenemos conexión? ¿Cómo se transformará la ciudad por el Internet de las cosas? ¿Quién manejará los datos (bigdata/fastdata) y la información que vamos dejando por nuestras conexiones? ¿Quedarán espacios libres de emisiones radioeléctricas? ¿Continúa la vida en el espacio hertziano una vez muertos en el mundo real?

Depositamos éstas y otras cuestiones en creadores y artistas, capaces de generar obras que vuelvan a conectar a los espectadores con un poco de esta complejidad, ampliando las perspectivas y obligando al público a darse cuenta de que las tecnologías, a menudo, tienen implicaciones y posibilidades más allá de los usos utilitarios por las que son conocidas. Terminamos este artículo, con el anhelo de que la investigación presentada sirva a otros investigadores y artistas (presentes y futuros) que tienen que lidiar y trabajar con aquellas manifestaciones y tecnologías que aún no han sido validadas como arte.

\section{Bibliografía.}

AlsinA, Pau (2007): Arte, ciencia y tecnología. Editorial UOC. Barcelona.

CASTELLS, Manuel (1996): La sociedad red. Volumen 1ํ. La era de la información: economía, sociedad y cultura. Alianza Editorial. Madrid.

COMUNICACIÓN MÓvIL y nuevo sentido de los lugares. Una crítica sobre la espacialización en la Cibercultura, en Inclusiva-net, \#2, Redes digitales y espacio físico. Medialab Prado, Madrid, p. 29.

DebAtTY, Régine (2011). New Art/Science Affinities, The Miller Gallery, Carnegie Mellon University y STUDIO. Pittsburgh. 
DunNE, Anthony y Raby, Fiona (2001): Design Noir: The Secret Life of Electronic Objects. Birkhauser. Basel.

DunNE, Anthony (2005). Hertzian Tales: Electronic Products, aesthetic experiencie and critical design. MIT Press. Cambridge, Massachussets.

ECHEVERRÍA, Javier (1999): Los Señores del Aire: Telépolis y el Tercer Entorno. Destino. Barcelona.

GIBSON, William (2006): Neuromante (Neuromancer). Minotauro. Barcelona.

IGÉS, José (1997): “La ciudad resonante”, en Fisuras de la cultura contemporánea. № 5, Las ciudades inasibles, Madrid, pp. 60-78.

ITO, Toyo (2000). Escritos. Coat. Murcia.

ITO, Toyo (2006): Arquitectura de límites difusos. Gustavo Gili. Barcelona.

KAC, Eduardo (2010): Telepresencia y bioarte: interconexión en red de humanos, robots y conejos. CENDEAC, Murcia.

LEMOS, André (2008): “Medios locativos y territorios informativos. Comunicación móvil y nuevo sentido de los lugares. Una crítica sobre la espacialización en la Cibercultura", en Inclusiva-net, \#2, Redes digitales y espacio físico, Medialab Prado, Madrid.

LÉVY, Pierre (1999): ¿Qué es lo virtual? Paidós. Barcelona.

Mitchell, William J. (1995): City of bits. Space, place and the infobahn. The MIT Press. Cambridge, Massachussets.

Mitchell, William J. (2003): M++. The Cyborg Self and the Networked City. The MIT Press. Cambridge, Massachussets.

NovaK, Marcos (1993): “Architetture liquide nel ciberspazio", en Cyberspace, Muzzio, Padova, Pág. 233 -265.

STERLING, Bruce (1992): The Hacker Crackdown: Law and Disorder On the Electronic Frontier. Spectra Books. New York.

VIRILIO, Paul (1991): The Lost Dimension. Ed.Semiotexte. New York. 


\section{Páginas Web y documentos online.}

http://alt1040.com/2012/05/inteligencia-artificial-las-ciudades-comoorganismos-vivos Documento online: Komninos, Nicos. "Inteligencia Artificial: Las ciudades como organismos vivos", Mayo de 2012.

http://espacio.fundaciontelefonica.com.ar/wp-

content/uploads/descargas/1368107281-Nomadismos.pdf. Documento online: Lemos, André. "Cultura de la movilidad". En Nomadismos Tecnológicos. Web consultada el 03/05/16

http://hackitectura.net/osfavelados/txts/sci_fi_geographies.html, Web consultada el 20/01/15

http://rixc.lv/waves/, Web consultada el 23/02/15

http://www.artfutura.org/02/05haque.html, Web consultada el 02/02/15

http://www.artssantamonica.cat, Web consultada el 23/02/15

http://www.cccb.org/es/actividades/ficha/la-conquista-invisible/218343, Web consultada el 23/02/15

http://www.haque.co.uk/papers/hardsp-softsp-open-so-arch.PDF, Web consultada el 02/02/15

http://www.hmkv.de/_en/programm/programmpunkte/2008/Ausstellungen/

Waves.php, Web consultada el 23/02/15

http://www.kunstverein-cuxhaven.de/ohne_schnur/englisch/index2.htm,

Web consultada el 23/02/15

http://www.ninaczegledy.net/?id=11, Web consultada el 24/02/15

http://www.smartcityexhibition.it/it/node/493. Entrevista a Nicos Komninos:

"The smart city is grounded on collective intelligence", sitio digital SmartCityExhibition., Web consultada el 23/02/15

http://www.tate.org.uk/context-comment/vídeo/softspace-contemporaryinteractive-environments, Web consultada el 06/05/13 
http://www.zemos98.org/festivales/zemos988/reclaim/intro.htm, Web consultada el 23/02/15

https://idus.us.es/xmlui/bitstream/handle/11441/26693/Una\%20historia\%20del \%20Arte\%20de\%20Internet.pdf?sequence=1. Documento online: Greene. R. "Una historia del Arte de Internet". Original publicado como "Web work a history of internet art" en ARTFORUM International, nº 9 May 2000, Págs. 162-167, 190. Web consultada el 02/12/16 